\title{
Le comportement sexuel des adolescents à Bangui (RCA)
}

\author{
Frédéric KOBELEMBI \\ Démographe, Chargé de Suivi évaluation \\ au Secrétariat Technique du Comité National \\ de Lutte contre le VIH/SIDA, \\ Bangui République Centrafricaine
}

\section{Résumé}

La sexualité des adolescents est devenue un sujet de préoccupation de santé publique, avec l'extension du SIDA au cours de ces dernières années, particulièrement en milieu urbain. Quels sont les déterminants des pratiques sexuelles des adolescents? Pourquoi certaines pratiques peuvent-elles être qualifiées de comportements à risque? Dans quelles mesures les politiques de santé publique pourront-elles résoudre les problèmes liés à ces pratiques sexuelles? C'est pour apporter des éléments de réponse à ces questions que l'enquête sur le comportement sexuel a été menée auprès de 806 adolescents filles et garçons dans la ville de Bangui. Huit discussions de groupes ont été également organisées avec ces adolescents et leurs parents. Les principaux résultats sont les suivants : la première expérience sexuelle est vécue entre 15 et 16 ans respectivement chez les filles et chez les garçons; près de la moitié des enquêtés sont sexuellement actifs une fois par semaine; plus de quatre adolescents sur cinq connaissent les conséquences néfastes des rapports sexuels précoces (grossesses non désirées, MST, SIDA); un adolescent sur deux a eu son dernier rapport sexuel protégé. Les résultats de l'analyse multivariée montrent que les chances d'utilisation des préservatifs sont plus réduites chez les moins jeunes (12-14 ans) que chez les plus âgés (15-19 ans), tandis que le milieu de socialisation de l'enfant (Bangui ou autre) n'a aucun effet significatif sur l'utilisation des préservatifs. La fréquentation des structures d'éducation sexuelle, la communication en matière de sexualité avec les parents et le multi partenariat sont les principaux déterminants de l'utilisation des préservatifs.

\section{Introduction}

La sexualité des adolescents est un problème de santé publique dans le monde. La République Centrafricaine, à l'instar de nombreux pays, est confronté à ce problème. Les statistiques sont suffisamment alarmantes et justifient la prise de conscience. Selon les rares données statistiques disponibles, on note que la proportion de grossesses contractées en milieu scolaire varie entre 9 à $14 \%$. Aussi, une enquête réalisée à l'Unité de Dépistage Anonyme de VIH/SIDA à Bangui en 1997 relève que dans la tranche d'âges 15 à 24 ans, une fille court cinq fois plus de risque d'être infectée par le VIH qu'un garçon (J. Sehonou).

Les autorités centrafricaines reconnaissent que des mesures spécifiques peuvent aider les jeunes à éviter une grossesse non désirée et précoce, à limiter le recours à l'avortement, à prévenir la diffusion des infections sexuellement transmissibles comme le VIH/SIDA. Cette préoccupation pour le comportement sexuel des adolescents en Centrafrique est sous-tendue par 
les facteurs suivants (i) la croissance démographique qui a pour corollaire l'augmentation de la proportion de la population de moins de 20 ans; le processus d'urbanisation et de modernisation qui a gravement perturbé l'environnement traditionnel des adolescent(e)s et leur lien avec le reste de la société; (ii) les rites traditionnels marquant le passage de l'enfance au statut d'adulte qui ont largement été bouleversés; (iii) l'immaturité physique qui expose les adolescents à des risques si la sexualité ou la reproduction commence trop tôt. Ces trois éléments ont montré que les adolescents représentent un groupe ayant des particularités psychologiques, sociologiques et physiologiques qui méritent une attention soutenue.

Les études menées jusqu'alors en Afrique et plus particulièrement en Centrafrique ont porté pour l'essentiel sur les niveaux d'activité sexuelle, les faibles niveaux d'utilisation de la contraception et les conséquences éventuelles de la sexualité précoce chez les adolescents en milieu scolaire. Rares sont les recherches orientées vers l'identification des déterminants du comportement sexuel des adolescents.

C'est dire que de nombreuses interrogations n'ont pas encore trouvé des réponses satisfaisantes. Quels sont par exemple les aspects et les déterminants des pratiques sexuelles des adolescents? Pourquoi certaines pratiques peuvent-elles être qualifiées de comportements à risque ? Dans quelle mesure la politique de santé peut t-elle répondre à ces problèmes ? Toutes ces questions demeurent sans réponse et constituent par conséquent autant de problématiques. Nous avons choisi de les aborder. Nous nous proposons en somme, à partir de cette étude centrée sur les adolescents de la ville de Bangui, de tirer des enseignements qui pourront alimenter les stratégies des gestionnaires des programmes de santé sexuelle. Le choix du sujet se justifie par les conditions dans lesquelles vit la majeure partie des jeunes centrafricains de la capitale Bangui, conditions qui les empêchent de mener une vie suffisamment saine et de contribuer ainsi au développement de la nation. Notre intérêt pour le problème que pose la sexualité des adolescents a été suscité par les craintes et des menaces qu'ils font peser sur la santé. Pour cerner ce problème, le présent article se propose de rechercher les déterminants du comportement sexuel et d'inventorier les mesures les plus appropriées pour remédier aux éventuels problèmes répertoriés. Pour saisir les attitudes sexuelles des adolescents en Centrafrique, nous avons limité le champ de l'étude à la ville de Bangui.

Deux principales techniques de recherche ont été utilisées dans ce travail. La première technique a consisté à rassembler des informations documentaires sur la sexualité des adolescents; la seconde à mener une enquête auprès d'un échantillon d'adolescents. 


\section{Cadre théorique}

\section{Définition des concepts de base}

Pour une bonne compréhension des analyses présentées, les concepts d'adolescent et de comportement sexuel méritent d'être définis au début de cette étude.

\section{a) L'adolescence}

L'adolescence est une période pendant laquelle (i) l'être humain passe du stade de la première apparition des caractères sexuels secondaires à celui de la maturité sexuelle; (ii) il acquiert des structures psychologiques et les méthodes d'identification qui transforment l'enfant en adulte; (iii) une transition se réalise entre le stade de dépendance sociale et économique totale à celui de l'indépendance relative.

Cette identification prend en compte quatre étapes de l'adolescence :

- l'éveil sexuel vers 13-15 ans ;

- les premières relations sexuelles vers 14-17 ans ;

- le rôle sexuel vers 16-19 ans ;

- le choix d'un rôle déterminant dans la société vers 18-25 ans.

Le concept d'adolescence recouvre plusieurs dimensions: biologique, démographique, sociale, psychologique, juridique, économique ; il n'est par conséquent pas étonnant que les définitions utilisées diffèrent selon les chercheurs. L'absence d'une définition univoque de ce concept rend ainsi difficile la détermination d'une période stable de la vie à laquelle s'appliquerait l'adolescence.

Dans le cadre de notre étude et pour les besoins de l'analyse, nous convenons de définir l'adolescence sociale comme la période de vie de l'homme allant de 12 à 19 ans.

\section{b) Le comportement sexuel}

Pour certains auteurs, «le comportement sexuel désigne pour chaque individu, une configuration qui comprend un répertoire de pratiques sexuelles, un répertoire de scénarios et un répertoire de significations " (Bajos et al, 1993,:33). Les pratiques sexuelles désignent les types de contacts corporels, non nécessairement mutuels, liés à l'excitation sexuelle d'au moins une personne; un scénario est une série de pratiques sexuelles, réalisées dans un contexte donné avec un partenaire donné. La signification ou la représentation désigne les valeurs et fonctions attribuées, consciemment ou non, à l'activité sexuelle (Giami, 1991, 1215). 
On conçoit alors que le comportement sexuel peut recouvrir un vaste champ pouvant comprendre le fantasme, le voyeurisme, la masturbation solitaire ou en groupe, les rapports sexuels avec ou sans pénétration, les rapports péno-vaginaux ou péno-anaux, les rapports génito-oraux, les rapports protégés ou non. Aussi, tous les comportements sexuels, y compris ceux des adolescents, ne se traduisent pas nécessairement par des pratiques à risque de maladie sexuellement transmise ou de grossesse non désirée.

\section{Approches explicatives du comportement sexuel des jeunes}

De la littérature traitant de la question, il se dégage plusieurs approches dans l'explication de l'activité sexuelle des adolescents. Nous en retiendrons cinq principaux dans la suite de notre travail: l'approche biologique, l'approche socioculturelle, l'approche socio-économique, l'approche institutionnelle et l'approche de genre.

\section{Approche biologique}

L'approche biologique postule que l'activité sexuelle des jeunes résulte d'un mécanisme purement biologique et donc naturel. Pour Freud (1905) cité par Rwenge (1995), les types de comportements sexuels sont le résultat d'un vif désir sexuel. Selon cet auteur, l'activité sexuelle serait le résultat d'une pulsion biologique que l'individu chercherait à satisfaire à n'importe quel prix, directement ou indirectement. Les contraintes extérieures imposées par la société seraient le cadre approprié défini par celle-ci pour réglementer toute activité sexuelle. Il ne serait pas superflu de conclure à l'universalité de l'expérience sexuelle, les variations n'étant dues qu'aux normes sociales qui régissent l'activité sexuelle. Emboîtant le pas à Freud, Bancroft et Skakkebaek en 1978, Udry et al en 1986 ont montré que l'activité sexuelle est déterminée par un mécanisme hormonal naturel, donc biologique.

Ces résultats sont davantage confortés par les travaux de Beach qui a montré en 1974 que l'activité sexuelle augmente avec le niveau des hormones à l'adolescence. De ce point de vue, on pourrait conclure à une universalité de la pratique des rapports sexuels dont les seules limites seraient les contraintes extérieures imposées par la société.

Les sociologues se sont élevés contre cette approche, lui reprochant de "désocialiser" en grande partie l'activité sexuelle en faisant passer pour secondaire la construction sociale et culturelle de l'activité sexuelle. Pour eux, ces relations ne devraient pas être extraites du contexte social dans lequel elles se déroulent.

\section{Approche socioculturelle}

Pour les sociologues, l'engagement ou non des jeunes dans l'activité sexuelle dépend largement du milieu dans lequel ils vivent, des perceptions que la société a des relations sexuelles, du rôle assigné à l'activité sexuelle, des 
normes qui la régissent. Ils estiment que les comportements sexuels sont déterminés par les normes et les valeurs socioculturelles en vigueur. Une modification de ces normes et valeurs aura certainement une incidence sur le comportement sexuel des individus qui composent le corps social. De fait ils rejettent l'approche biologique comme dans ces propos de Twa-Twa (1997) : "The social science proposes that libido or sexual motivation, is generated purely by social processes that fill the culture with the message that sex is highly satisfying and identify the pubertally developed adolescent as an attractive and sexual participant. Hormones are relegated to the single role of producing pubertal development, which is socialy interpreted as the signal by the female adolescent and those arround her that she is ready to enact the social "script» of sexual behaviour».

Ils soutiennent que les comportements sexuels et les circonstances dans lesquelles se déroule l'activité sexuelle sont déterminées par les normes et les valeurs socioculturelles en matière de sexualité.

La théorie générale de modernisation tente d'expliquer les tendances sexuelles actuelles des jeunes africains. Selon elle, l'adoption de nouveaux comportements sexuels par les jeunes pourrait trouver son explication dans l'influence de la modernisation qui sape progressivement les valeurs traditionnelles en matière de sexualité.

Certains auteurs parmi lesquels Gagnon et Simon (1973), Hoffert et Hayes (1987), Bozon (1994) soutiennent que la motivation sexuelle est générée par des processus sociaux, par l'environnement et le cadre de vie. Ils n'accordent pas un grand rôle aux hormones qui selon eux n'interviennent que dans la croissance et la maturation des organes sexuels secondaires. Les comportements sexuels pour ainsi dire sont déterminés par les normes et valeurs socioculturelles en matière de sexualité.

\section{Influence de la modernisation et diminution du contrôle social des aînés}

Dans la société traditionnelle, le développement des jeunes se fait à l'intérieur de la structure familiale. Toute leur éducation et leur apprentissage à la vie sont assurés par les aînés ou les parents à qui ils doivent respect et obéissance. Le contrôle social est assuré en permanence (Kouton, 1992). Vis-à-vis de leurs fils, les parents n'estiment s'être acquittés de leur devoir qu'après leur avoir donné des parcelles pour l'implantation de leurs maisons, et après leur avoir trouvé une épouse. Pour les filles, les parents visent le mariage (Dongmo, 1981). Dans ce contexte de contrôle social serré, l'individu est géré par la communauté qui lui dicte jusqu'à ses conduites individuelles et interindividuelles. L'adolescent n'a pas le pouvoir de décision. Ce sont les parents qui concluent les unions entre fils et filles de la communauté, sans la moindre référence à leurs sentiments. De cette façon, l'activité sexuelle, même si elle est précoce (stratégie de la plupart des sociétés africaines traditionnelles pour obtenir une progéniture élevée), est dirigée par les aînés. Aux jeunes filles pubères, il est enseigné les astuces 
pour tenir un mari et faire honneur à son époux. Les valeurs telles que la virginité prénuptiale, la chasteté, la soumission au mari sont enseignées aux jeunes filles. Aux garçons, l'accent est mis sur le sens des responsabilités et sur les rôles qui leur sont dévolus par la société.

Cette harmonie traditionnelle est ébranlée par l'introduction et la diffusion de la culture des colonisateurs. Les villes, pôles d'expérimentation de cette culture, jouent le rôle de relais entre le monde rural et le monde occidental. Aujourd'hui, l'individu n'est plus sous l'autorité du groupe vis-à-vis duquel il prend de plus en plus son indépendance.

"Economic development, urbanization and new gender roles have change the behaviour of young people in developing countries to a great extend... Migrations to cities, mass media, unemployment, and peer pressure are another set of social and economic changes that have help loosen family ties, break cultural boundaries, and encourage premarital relations. » (El-Tawila, 2000, p. 5).

Les valeurs de liberté et de démocratie qu'on enseigne aux jeunes leur donnent l'occasion de s'insurger contre l'autorité des parents ou des aînés. Les activités nouvelles contribuent davantage à soustraire les jeunes de l'autorité parentale. Selon Rwenge, "l'école et les nouvelles valeurs récréatives éloignent souvent les jeunes des adultes. En plus la séparation des sexes n'y est plus assurée. Toutes les nouvelles inventions récréatives telles que le cinéma, les soirées dansantes, le football... raccourcissent le temps que les jeunes passent sous le contrôle des parents ou dans ce cercle familial. » La conséquence de cette situation est le développement d'une activité sexuelle précoce chez les jeunes, fait observé dans plusieurs villes africaines.

La ville présente des caractéristiques particulières qui font d'elle un important centre de diffusion de la culture occidentale. Pour Gueye et al. (2000), "Incrising modernization and media exposure, along with a decline in the authority of parents and elders, have undermined the societal and cultural rules that formerly controlled and informed adolescents sexuality». Les villes du tiers monde en particulier connaissent une croissance très rapide. Cette augmentation rapide de la population sur un espace réduit engendre une certaine promiscuité.

Par ailleurs, certaines catégories de populations des villes ont un niveau de vie assez élevé pour s'offrir les nouvelles technologies de l'information et de la communication. Aujourd'hui le paysage audiovisuel camerounais foisonne de nombreuses chaînes de télévision nationales et internationales. Grâce à des "câblo-opérateurs"1 privés, beaucoup de ménages ont la

1 Les câblo-opérateurs sont des hommes d'affaires qui permettent aux ménages de recevoir des images des chaînes étrangères, occidentales notamment, moyennant le versement mensuel d'une somme variant généralement entre 5000 et 10000 francs CFA. 
possibilité d'obtenir les images venant des autres parties du monde, de l'Occident notamment. Si les médias peuvent permettre aux jeunes d'accroître leur connaissance et favoriser une sexualité saine, ils peuvent en même temps être à l'origine de dérives comportementales sur le plan sexuel. En effet, certains médias audiovisuels occidentaux, en raison de la mondialisation, "inondent" l'Afrique d'images indécentes. Ces chaînes "pornographiques" qui font l'apologie de l'activité sexuelle pré-maritale poussent les jeunes à s'engager dans l'activité sexuelle. Il s'installe une véritable tension entre l'apologie des relations sexuelles pré-maritales et l'effort des autorités ecclésiastiques pour la promotion des comportements sexuels responsables. C'est sans doute l'influence de ces médias qui est à l'origine de la modification de la signification de l'acte sexuel chez les jeunes. La recherche du plaisir est devenue la raison principale des rapports sexuels. Ainsi les jeunes sont de plus en plus exposés aux images des télévisions étrangères qui n'ont pas toutes à cœur de préserver les mœurs de ceux qui les regardent ${ }^{2}$. Cet extrait de Béat-Songué illustre parfaitement cette situation: «En ville, les adolescents se délectent plutôt de ce que leur offrent les films de nombreux clubs-vidéo. La sélection des films donne priorité aux films pornographiques qui sont consommés par les adolescents. Des romans du même type sont vendus aux adolescents dans les librairies de la rue et les librairies du "poteau », commercialisant de vieux livres d'occasion à vil prix sur les trottoirs des places publiques très fréquentées. » (Béat-Songué, 1998, p. 185).

L'accès à ces hauts lieux de perversité et de violence n'est conditionnée que par le paiement d'une modique somme de 25 à 100 francs CFA, ce qui est à la portée des jeunes. Par ailleurs, selon l'auteur, "l'imitation des modèles proposés par ces films en matière de sexualité s'ensuit, et ceci est réalisé en compagnie d'autres adolescents... Le but de l'expérimentation de la sexualité ainsi que sa pratique et son expression sont de plus en plus influencés chez les adolescents par ce qu'ils reçoivent de ces médias nouveaux. » En 1995 à Bamenda, Rwenge a constaté que les médias étaient un facteur déterminant de la primo-nuptialité, en tant qu'ils favorisent le relâchement des mœurs. D'autres auteurs ont confirmé cette hypothèse de la baisse du contrôle social sur la sexualité des jeunes (Ilinigumugabo, 1996; Mankamté, 1997). L'exposition des jeunes à ces médias d'"outre mer" est suivie d'un mimétisme presque complet. Il n'est donc pas étonnant que l'on assiste aujourd'hui à un recul des valeurs traditionnelles qui ont jadis gouverné les attitudes et les comportements des parents dans leur jeunesse. Ceux-ci sont désormais les témoins impuissants de nouveaux comportements que les jeunes affichent parfois avec ostentation. Les jeunes ont une nouvelle perception de l'activité sexuelle dont le rôle essentiellement procréateur est de plus en plus ignoré.

2 Certaines de ces chaînes ont parfois la triste célébrité de diffuser des images à la limite de l'indécence. 
Beaucoup d'auteurs considèrent cette baisse du contrôle social comme responsable de la recrudescence de l'activité sexuelle précoce chez les jeunes. "The removal of sexuality from the control of the community resulted in individual decisions about when, where, with whom and for what purpose to have sexual intercourse." (Meekers, 1994). Gage et Meekers illustrent cette situation en présentant les résultats d'une étude réalisée au Kenya où $60 \%$ des jeunes interrogées ne pensent pas que les normes traditionnelles restrictives pour les relations sexuelles pré-maritales et extra-maritales puissent encore être applicables dans la société contemporaine. La virginité avant le mariage n'est plus d'une grande importance. Ces propos relevés par Calvès au cours des discussions de groupe auprès des filles à Yaoundé en est une illustration : «Les filles ne peuvent pas se permettre d'arriver la nuit de noce toute bête, ne sachant rien » (p. 166).

En somme l'influence de la diffusion des modes de vie occidentale, notamment sur le plan de la sexualité, est un facteur primordial d'incitation à l'engagement dans les rapports sexuels chez les jeunes dans un contexte où le contrôle des parents et des aînés perd de son importance.

\section{Influence de la scolarisation}

La baisse du contrôle social ou des parents est aussi à mettre à l'actif des effets négatifs de la scolarisation. Celle-ci écarte les enfants de leur milieu familial pendant plusieurs heures par jour. Le temps consacré à leur éducation par les parents se trouve ainsi réduit (Rwenge, 1995). Cette absence prolongée du cadre familial diminue le contrôle physique et psychologique des parents sur leur progéniture. Bien plus, en enseignant les nouveaux modes de pensée tels que la démocratie et la liberté, les droits de l'enfant, la scolarisation donne aux jeunes la possibilité de contester certaines valeurs et normes qu'ils estiment appartenir à l'ancienne époque. Parlant de la diminution du contrôle social sur les jeunes, Meekers (1997) estime que: "This change can be attributed to the fact that educated youth now obtain knowledge from books which can be used to challenge the wisdom of the older generation. »

Ces auteurs sont parvenus aux conclusions selon lesquelles l'instruction est positivement corrélée à l'utilisation des préservatifs alors que ce sont les personnes plus instruites qui s'engagent plus fréquemment dans des rapports sexuels occasionnels.

En contribuant à l'augmentation de l'âge au premier mariage, la scolarisation est aussi responsable de l'augmentation de la prévalence de l'activité sexuelle chez les jeunes. "The litterature on African family formation suggests that age at marriage is rising in many African societies, especially among the better educated and urban segments of the population. " (Meekers, 1994, p. 1). Dans le même temps, l'âge au premier rapport sexuel diminue, aboutissant ainsi à une augmentation substantielle de la période d'exposition au risque de s'engager dans les rapports sexuels. «Les études relatives au début des 
relations sexuelles et au moment du mariage en Afrique subsaharienne documentent une hausse de l'activité sexuelle prénuptiale sans cohabitation. » (Magnani, 1995) ${ }^{3}$. Tandis que l'âge au moment des premières règles se réduit et que celui du mariage augmente, la période entre les deux s'allonge, accroissant ainsi la période des risques d'activité sexuelle prénuptiale (Sushuela et Renee, 1997; Meekers, 1998). Cette évolution purement démographique pourrait bien expliquer la recrudescence de l'activité sexuelle chez les jeunes.

Toutefois, ce procès négatif de la scolarisation est tempéré par d'autres auteurs pour lesquels l'instruction retarderait l'occurrence des premiers rapports sexuels chez les filles (Ilinigumugabo et al. 1996 ; Rwenge, 1995 ; Meekers et Calvès, 1997).

\section{Communication timide entre jeunes et parents et pression des pairs}

$\mathrm{Au}$ Cameroun, la sexualité semble être encore un sujet tabou entouré de mystères. Les parents répugnent à aborder un tel sujet avec leurs enfants (Njoh, 1996). L'éducation sexuelle en famille est presque inexistante (Rwenge, 1995 ; Kouintche et Tagne, 1998). Il en résulte que beaucoup de jeunes filles parviennent à la maturité sexuelle sans bonne connaissance sur le fonctionnement de leur appareil reproducteur, sans aucune connaissance sur la sexualité (Ilinigumugabo et al. 1996). Leke (1998, p. 281) écrit : "Selon les études, moins de $30 \%$ des Africaines connaissent leur période féconde. » Ce manque de communication est illustré par la catégorie des personnes avec lesquelles les jeunes s'entretiennent des MST/VIH-Sida. Le plus souvent, c'est avec leurs amis que les jeunes abordent cette discussion. Les jeunes subissent donc l'influence de leurs amis et ce d'autant plus qu'ils ne reçoivent aucune autre information de la part de leurs parents (Calvès, 1998 ; Songue, 1998). Face à la pression de leurs pairs qui les invitent à avoir des relations sexuelles comme eux, les filles n'ont parfois pas la force de résister. En milieu urbain camerounais où les relations sexuelles avant le mariage sont devenues la règle, "les normes sociales encouragent si fortement la sexualité des jeunes adolescents que, passé un certain âge, celles qui sont encore vierges se sentent plutôt marginalisées. » (Calvès, 1998). Cette situation favoriserait chez les jeunes une sexualité à risque (précocité des rapports sexuels et rapports sexuels occasionnels), car ceux-ci n'ont pas toujours les informations nécessaires ou reçoivent de la part de leurs amis des informations erronées.

3 Magnani et al., 1995, "Men Marriage and Fatherhood in Kinshasa, Zaire », in International Planning Perspectives, $\mathrm{n}^{\circ}$ 21, p. 19-25. 


\section{La perception du condom comme un objet chargé de préjugés}

$>$ Condom et réduction du plaisir sexuel

L'époque où on liait l'activité sexuelle au mariage est révolue. Personne n'y croît plus aujourd'hui car de plus en plus les rapports sexuels n'ont plus pour seul but la procréation. Avec l'évolution du temps, la recherche du plaisir est devenue l'une des raisons principales de l'acte sexuel. Ceci est $d^{\prime}$ autant plus vrai que la plupart des jeunes ayant eu un enfant n'ont jamais désiré l'avoir. Du coup, tout élément qui entraîne ou est supposé entraîner une réduction du plaisir est immédiatement rejeté. Le condom, quoiqu'il protège contre les IST/VIH-Sida, en s'intercalant entre l'homme et la femme au cours du rapport sexuel, réduirait le plaisir tiré de l'acte sexuel. C'est là l'une des raisons qui sont évoquées pour expliquer la faible utilisation des préservatifs masculins par les populations africaines et notamment par les jeunes.

$>$ Le préservatif : une menace permanente de rupture de relation

Si le préservatif masculin protège contre les IST/VIH-Sida, son utilisation n'est pas évidente et dépend non seulement de son coût mais aussi de l'image et de la représentation qu'il véhicule parmi les populations. De cette image et de cette représentation dépendent l'acceptation et l'utilisation de «corps étranger». Dans beaucoup d'unions, l'exigence du port du préservatif est source d'accusation et de suspicion de l'un des partenaires. Ainsi, l'homme qui porte le préservatif soupçonne indirectement sa partenaire de mener une vie sexuelle indisciplinée ; il en est de même de la femme qui exige de son partenaire l'utilisation du préservatif. Le condom est ainsi devenu synonyme de méfiance. Cette méfiance est à son tour un facteur de l'érosion de la confiance entre les partenaires et une menace de rupture d'union. C'est ainsi que les partenaires qui tiennent à leur union renonceraient volontiers à un recours aux préservatifs (Bond and Dover, 1997). De même chez les jeunes filles qui tiennent à ne pas perdre leur petit ami et chez les jeunes garçons qui ne veulent pas vexer leur petite amie, on préfère ne pas s'embarrasser de condom. Pour illustrer cette relation entre la confiance et l'utilisation des moyens de protection, l'étude de l'IRESCO parmi les jeunes de Yaoundé et de Douala a relevé que le condom a été d'autant moins utilisé que le lien avec le partenaire était étroit : 9,5\% de jeunes ont utilisé le condom avec leur partenaire habituel 4 , 57,3\% avec un partenaire sexuel occasionnel et $98 \%$ avec un partenaire commercial.

\section{Approche économique}

L'approche économique se fonde sur les deux postulats de base de l'économie à savoir l'individualisme méthodologique et la rationalité

4 Il s'agit dans la majorité des cas d'un partenaire sexuel régulier et non de conjoint au sens d'époux ou d'épouse. 
économique. Cette approche stipule que l'activité sexuelle des jeunes et les comportements qui la régissent sont le résultat d'un calcul rationnel de leurs auteurs. Ainsi donc, les jeunes qui s'engagent dans l'activité sexuelle et adoptent certains comportements ne le font qu'à la suite d'une décision bien réfléchie, en vue d'atteindre des objectifs bien précis d'ordre économique et social. "...Unmarried teenagers use sexual relations and pregnancy to accomplish certain goals. For example, sexual relations may have economic benefits or be a step towards marriage. » (Meekers, 1994). Cherlin et Riley (1986) cités par Meekers en 1994 ont distingué deux catégories d'objectifs poursuivis par les jeunes : l'objectif économique et l'objectif social.

\section{L'activité sexuelle : un moyen d'améliorer ses conditions économiques}

Certains adolescents ont des rapports sexuels contre une gratification financière ou des cadeaux qui leur permettent de satisfaire leurs besoins matériels et financiers. L'activité sexuelle devient alors une stratégie de survie, un moyen de se mettre à l'abri du besoin matériel et financier. Il s'agit des filles qui dans l'incapacité de satisfaire leurs besoins financiers sont obligées de commercialiser leur sexe aux hommes de situation financière aisée (Calvès, 1996 ; Ilinigumugabo et al., 1996). " Si l'argent ne constitue pas une motivation importante pour la première expérience sexuelle, il constitue toutefois par la suite une composante majeure de leur vie affective.»(Calvès, 1998, p. 167). Ainsi les avantages financiers découlant de la multiplicité des partenaires sont cités par un nombre important de jeunes femmes âgées de 20 ans et plus. Au cours de son enquête, Calvès a relevé que $47 \%$ d'entre elles affirment qu'elles ont plusieurs partenaires afin de satisfaire leurs besoins financiers. Cette monétarisation des rapports sexuels se fait non seulement avec des partenaires plus âgés et plus riches (relations du type "sugar dadies" ou encore "sponsors") mais aussi avec leurs jeunes copains de qui elles exigent des faveurs similaires en échange de relations sexuelles. Dans les discussions de groupe, il ressort que le phénomène de "sugar dadies" fait aussi partie des stratégies pour la recherche d'un emploi car «...un parrain qui a souvent une bonne situation professionnelle et de nombreux contacts, peut rendre également des services à sa partenaire, comme l'aider à trouver un emploi. » (Calvès, 1998, p. 165).

Les filles ne sont cependant pas les seules à recourir aux relations sexuelles de ce type pour satisfaire des besoins financiers. Les garçons sont eux aussi plus ou moins impliqués dans cette activité sexuelle rétribuée. En effet, certaines femmes un peu plus âgées se proposent d'aider financièrement des jeunes hommes en contrepartie des rapports sexuels que ces derniers auront avec elles (relations du type "sugar mummies"). Bien plus, la perversité pousse certains jeunes à avoir des rapports homosexuels avec des hommes plus âgés contre une gratification financière (Kaptue, 1998). Rwenge a relevé en 1995 que la précarité peut aussi expliquer les rapports sexuels occasionnels des hommes car ceux-ci n'ont pas assez de moyens pour 
entretenir une partenaire régulière. Dans ce cas, ils se contenteraient de relations sexuelles occasionnelles peu stables et peu coûteuses. Ces données montrent bien que les conditions économiques peuvent avoir une influence sur le comportement sexuel des jeunes.

Relevons que la situation économique peut agir dans un double sens : en cas d'insuffisance des moyens financiers, les jeunes filles auront tendance à se prostituer et les jeunes garçons à avoir des rapports sexuels occasionnels ; dans le cas de suffisance des moyens financiers par contre, les jeunes filles adopteraient des comportements sexuels plus sains alors que les garçons s'engageraient volontiers dans le multipartenariat.

En somme, les contraintes économiques auxquelles font face les jeunes peuvent les pousser à se compromettre dans des relations sexuelles rétribuées. Cette dépendance économique enlève aux jeunes qui en sont les victimes le pouvoir d'exercer un contrôle sur les modalités du déroulement de l'acte sexuel. Ils peuvent ainsi être contraints à avoir des rapports sexuels non protégés, selon la volonté du partenaire qui exerce sa domination.

\section{Approche institutionnelle}

Cette approche est basée sur l'idée que l'environnement institutionnel en matière d'activité sexuelle aurait une influence sur les comportements sexuels des jeunes. Il s'agit de déterminer la manière dont les institutions politiques gèrent les questions relatives à la sexualité ainsi que les stratégies qu'elles mettent en œuvre pour faire face aux IST/Sida. Cette approche accorde une grande importance aux politiques, aux programmes et aux lois en matière de sexualité relatifs aux comportements sexuels des adolescents. Si jusqu'à une date très récente, l'Afrique n'avait pas une tradition de lutte contre les IST, force est de constater que depuis la découverte du VIH/Sida, des efforts significatifs des gouvernements dans ce sens ne passent pas inaperçus.

L'attitude et l'implication des autorités dans la lutte contre les IST/Sida peuvent avoir une grande incidence sur la propagation de ces maladies parmi les jeunes. On peut imaginer l'impact négatif que peut avoir la légalisation de la prostitution sur le comportement sexuel des jeunes : ceuxci pourraient adopter un comportement liberticide sur le plan sexuel. Une loi légalisant la pratique de l'avortement est un couteau à double tranchant : en même temps qu'elle peut être d'un apport certain sur le plan de la santé de la reproduction, elle peut aussi inciter les jeunes à avoir une activité sexuelle intense et à risque.

La politique gouvernementale en matière de services de santé de la reproduction (SR) peut aussi avoir un impact sur le comportement des adolescents. Lorsque les programmes de population et les services sanitaires sont davantage dirigés vers les adultes que vers les groupes spécifiques des adolescents, ceux-ci se retrouvent moins bien informés des conséquences 
d'une sexualité incontrôlée. L'absence des structures sanitaires spécialisées dans les réponses aux problèmes des jeunes peut accroître leur ignorance des dangers qu'ils courent dans leur activité sexuelle. En revanche, la dissémination des services de SR dirigés spécifiquement vers les jeunes leur permettrait d'être mieux informés des questions relatives aux risques liés à l'activité sexuelle.

Dans nombre de pays africains, la législation sur les mariages est inexistante (Rwenge, 1999); là où elle existe, elle est le plus souvent favorable aux mariages précoces. De plus, elle pêche souvent par une grande imprécision qui rend son application difficile. En Centrafrique, l'âge minimum au mariage est fixé à 18 ans pour les jeunes filles et 20 ans pour les jeunes garçons. Malheureusement, lorsqu'elles existent, ces lois ne sont pas toujours respectées par certains parents conservateurs qui continuent à conclure le mariage de leurs filles sans leur consentement.

\section{L'approche émergente du genre}

C'est l'approche utilisée par l'Organisation mondiale de la santé (OMS) à travers le projet Santé familiale et prévention du Sida (SFPS). Les inégalités que l'on observe entre les sexes ainsi que les rapports qui en découlent sont des constructions sociales. Ces rapports sont connus sous le terme de «rapports de genre». L'approche de genre est fondée sur l'idée selon laquelle la réduction de l'écart entre les pouvoirs dévolus à chaque sexe par la société permettra à la femme de participer plus efficacement à la prise des décisions, notamment celles relatives à la santé de la reproduction. Cette approche vise le renforcement du pouvoir des femmes dans tous les domaines. Dans le domaine de la sexualité, l'approche du genre stipule qu'en raison de son faible pouvoir de décision, la femme ou la jeune fille n'a aucun contrôle ou alors n'a qu'un contrôle limité sur sa sexualité. Les rapports de genre en Afrique étant fortement inégalitaires, les femmes subissent la volonté des hommes à qui le rapport de force est favorable. Entre les jeunes, on pouvait bien s'attendre à une situation de plus grande compréhension entre les partenaires mais le problème demeure. L'écart d'âge entre les partenaires renforce aussi cette inégalité (les hommes plus âgés contractent très souvent des rapports sexuels avec des filles beaucoup plus jeunes).

L'adoption des comportements sexuels sains (utilisation des préservatifs) doit résulter d'une concertation entre les deux personnes en présence. Malheureusement, cette négociation n'est pas toujours de règle à cause du rapport de genre déséquilibré. Meekers et Megan relèvent que «Sexual risk reduction tends to be more difficult for women, as they do not use condom in isolation but negociate their use with their partners. " Beaucoup d'auteurs documentent des rapports inégalitaires entre les hommes et les femmes en Afrique dans les négociations sur les modalités de déroulement de l'acte sexuel. Ces rapports inégalitaires qui prennent leur source dans les rapports de genre se prolongent parmi les jeunes. On aboutit ainsi à des rapports de 
domination où les jeunes hommes peuvent dicter leur volonté sur la façon dont doit se dérouler l'acte sexuel. Les jeunes n'ont pas le pouvoir de demander, encore moins d'exiger de leur partenaire que le rapport sexuel soit protégé.

Cette situation de domination dégrade profondément l'image que les jeunes filles notamment peuvent avoir d'elles. "L'image de soi-même est la considération que l'on a de sa personne et la représentation que l'on fait de sa personne et de sa personnalité. » (Rwenge, 2001 inédit). Cette dégradation de l'image de soi-même agit négativement sur la perception de son autoefficacité, c'est-à-dire, la confiance qu'on a de son propre pouvoir de refuser certaines modalités des rapports sexuels ou de n'avoir que des rapports sexuels sans risque. Ainsi, "women who lack confidence in their ability to purchase condom and negociate their use tend to have a higher likelihood of engaging in unprotected intercouse. » (Meekers and Megan, 1997). Pourtant, une meilleure perception de l'auto efficacité (auto-efficacy) permettrait aux adolescentes de prendre conscience de la nécessité pour elles de se protéger ou de s'abstenir.

Les rapports inégalitaires, la mauvaise image de soi sont des facteurs psychosociaux qui déterminent parfois les comportements sexuels à risque. Dans l'étude des comportements sexuels des jeunes à Yaoundé et à Douala en 2001, près de $40 \%$ des filles estiment que l'usage des préservatifs dépend entièrement $d u$ garçon. C'est dire combien cette perception de l'auto efficacité affecte les comportements de prévention et l'adoption des comportements sexuels sans risque.

\section{Cadre conceptuel}

\section{Hypothèses de l'étude}

L'objectif de cette étude est de rechercher à déterminer les principaux facteurs qui expliquent les comportements en matière d'utilisation du préservatif pendant les rapports sexuels chez les adolescents non mariés, âgé de 12 à 19 ans, ayant déjà eu au moins un premier rapport sexuel et résidant à Bangui. Avant de présenter la méthode de régression et les variables retenues, il convient d'abord de rappeler les hypothèses de travail qu'on cherche à vérifier.

Ce cadre conceptuel explicite l'hypothèse fondamentale posée est la suivante : le comportement sexuel des adolescents est déterminé à la fois par les variables individuelles d'identification sociale et par les variables contextuelles (Hubert Gérard, 1989). Elle se décompose en deux hypothèses spécifiques de la façon suivante.

1- Les adolescents appartenant au groupe d'âges jeunes et dont le milieu de socialisation est autre que Bangui sont plus enclins aux rapports sexuels non protégés. 
2- L'utilisation par les adolescents des préservatifs pendant les rapports sexuels est fonction de la fréquentation des services d'éducation sexuelle, de la communication avec leurs parents, du nombre de partenaires sexuels et de la perception des risques des rapports sexuels non protégés.

\section{a) Définitions des quelques concepts clés}

\section{- Niveau de vie du tuteur ou du père de l'adolescent}

Il ressort de la littérature qu'il est difficile de cerner le concept de niveau de vie. Généralement, il est saisi à partir du revenu des parents ou de certains biens matériels dont disposent les parents. Il est reconnu qu'en Afrique, compte tenu de la structure des économies marquées par une forte proportion de la population active travaillant dans le secteur informel et des ménages vivant d'autosubsistance, le revenu n'est pas bien mesuré et ne peut donc pas bien rendre compte véritablement du niveau réel du ménage. Dans le cadre de notre étude, ce concept sera appréhendé à travers l'activité économique du père ou du tuteur de l'adolescent.

\section{- Milieu de socialisation}

Ce concept désigne le lieu où le jeune a passé son enfance c'est-à-dire la plus grande partie de ses douze premières années de vie. C'est donc le milieu où il a acquis un certain nombre de croyances ou d'habitudes, (durée de séjour à Bangui, changement d'école, environnement, etc.).

\section{- La communication sur la sexualité}

Cette variable composite renvoie à la notion d'Information, d'Education et de Communication (IEC), dans la mesure où les cours d'éducation sexuelle dispensés à l'école font appel à l'information et l'éducation. De même, au niveau du foyer, les parents peuvent compléter l'éducation scolaire en prodiguant de bons conseils aux adolescents pour qu'ils affrontent les réalités de la vie future de façon conséquente. Enfin, le dernier aspect concerne; le fait qu'ils discutent avec les parents, leurs pairs ou avec le personnel de santé va déterminer leur comportement.

\section{- Caractéristiques individuelles}

Variable composite renvoyant à l'ensemble des variables propre à l'individu. On trouve ici entre autres le sexe, l'âge, l'ethnie, la religion, le niveau $\mathrm{d}^{\prime}$ instruction, la pression des pairs etc.

\section{b) Spécification des variables utilisées}

\section{- Variables dépendantes}

Comportement sexuel

Nous distinguons deux types de variables dépendantes. Nous avons les variables de comportement sexuel à moindre risque et les variables de comportement sexuel à haut risque. Pour le premier cas, nous aurons des variables suivantes: la signification d'une relation sexuelle, avoir eu une 
relation sexuelle, le nombre de partenaire sexuel. Pour le second cas, c'est-àdire le comportement sexuel à haut risque la variable dépendante retenue est l'utilisation du préservatif au cours des relations sexuelles.

L'utilisation du condom ou du préservatif lors des relations sexuelles prend la valeur 1, et la non utilisation prend la valeur 0 .

Pour les adolescents qui n'ont pas encore eu des relations sexuelles, leur comportement sexuel est appréhendé à travers les variables relatives aux pratiques du comportement sexuel. La variable retenue est le fait d'avoir des relations sexuelles. La nature des variables dépendantes dépend du choix de la méthode statistique à utiliser.

\section{- Variables indépendantes}

Niveau d'instruction de l'enquêté

C'est le niveau atteint par l'individu dans le système de scolarisation formel. On peut le mesurer de plusieurs manières: soit par le nombre d'années passées à l'école, soit par le plus haut diplôme obtenu soit par la dernière classe achevée ou le cycle d'études. Nous définissons cette variable par le regroupement des individus selon les modalités suivantes: sans niveau, primaire, secondaire et supérieur.

\section{Religion}

C'est l'ensemble des pratiques et des rites propres à chacune des croyances. Elles constituent le véhicule d'un certain nombre de valeurs et normes qui régissent la vie des fidèles sur le plan comportemental, physiologique (Akoto, 1985). Les modalités retenues sont les suivantes: Protestant, catholique, musulman, animiste et sans religion.

Perceptions du risque

Pour adopter un comportement, l'adolescent évalue les conséquences des risques encourus. Pour saisir cette variable, nous avons retenu les variables suivantes: la connaissance des conséquences des rapports sexuels précoces et des moyens pour éviter les MST/SIDA. Pour la perception du risque, les modalités suivantes ont été retenues: Grossesses/MST/SIDA, le SIDA, la grossesse, la stérilité.

Fréquentation des structures d'éducation sexuelle des jeunes

La connaissance des structures d'éducation sexuelle des jeunes et la fréquentation des celles-ci peuvent déterminer le comportement sexuel des adolescents. Il s'agit ici d'un comportement sexuel à risque qu'il faut déterminer. Cette variable est saisie par le besoin d'informations sur la sexualité, la connaissance des structures d'éducation sexuelle et la fréquentation de celles-ci. 


\section{Méthodologie}

\section{Sources données}

En plus des statistiques sanitaires disponibles dans le centre scolaire et universitaire de Bangui et le rapport du Service de lutte contre les maladies sexuellement transmissibles et le Sida, nous avons collecté les données quantitatives à travers une enquête. Cette enquête a été réalisée auprès des adolescents de l'ensemble des huit arrondissements de la ville de Bangui. L'enquête par questionnaire est faite auprès d'un échantillon stratifié en fonction de deux variables essentielles, à savoir l'âge et le sexe. Compte tenu $\mathrm{du}$ fait que les questions liées à la sexualité demeurent un sujet tabou dans beaucoup de familles centrafricaines, nous ne pouvons pas utiliser la méthode de sondage probabiliste à plusieurs degrés comme la plupart des enquêtes démographiques le font. Nous avons plutôt utilisé la méthode des quotas, compte tenu des contraintes liées aux moyens financiers. La population cible de l'étude est constituée des adolescents de 12 à 19 ans résidant à Bangui.

\section{Les limites de l'enquête}

Les principaux biais rencontrés au cours de la présente étude concernent les biais de sélection dans la majeure partie des cas, le recrutement des participants s'est fait, pour raison de commodité, dans les établissements scolaires $(65,6 \%)$, dans les lieux de loisirs (22 \%), dans les lieux publics 11,5 $\%$, rarement dans la rue $(0,9 \%)$ et jamais à l'intérieur des maisons des intéressés.

\section{La méthode d'analyse multivariée}

Les modèles de régression logistique sont utilisés pour décrire les relations entre une variable dépendante à deux modalités et des facteurs de risques appelés variables indépendantes. Celles-ci peuvent être quantitatives ou qualitatives.

La variable dépendante de cette étude est l'utilisation de préservatif lors du dernier rapport sexuel. Si le dernier rapport sexuel est protégé, cette variable est codée 1 , sinon, elle prend la valeur 0 . Cette variable est régressée sur une série de variables dichotomiques ou poly-dichotomiques.

Il y a lieu de noter que la régression logistique utilise la méthode de maximum de vraisemblance pour estimer les paramètres du modèle. Cette méthode est essentiellement probabiliste, elle fournit des coefficients de régression $\mathrm{Bi}$ à partir desquels on calcule les risques relatifs (odds ratio). Pour mieux interpréter les résultats, on s'intéressera également aux risques relatifs. 
82 African Population Studies Vol.20 n²/Etude de la population africaine vol. $20 n^{\circ} 2$

Un risque relatif plus grand que 1 indique qu'il y a une plus grande probabilité que l'adolescent ait un rapport sexuel non protégé et un risque relatif plus petit signifie une probabilité plus faible que l'adolescent ait un rapport sexuel non protégé.

La stratégie d'analyse adoptée est une procédure pas à pas. Trois modèles caractérisent les interprétations des résultats multi-variés dont les variables indépendantes sont introduites par bloc. Le modèle de base comporte uniquement les variables relatives aux principales caractéristiques individuelles des adolescents. À ce modèle de base, il sera introduit dans la seconde étape, les variables sur les comportements sexuels des adolescents, ce qui constitue le modèle 2 . La dernière étape qui est notée modèle 3 , se caractérise par l'ajout au modèle 2, de nouvelles variables relatives à la communication en matière de sexualité chez les adolescents.

Cette procédure pas à pas a pour objectif de faire ressortir les effets nets sur la variable dépendante des autres variables quand on introduit certaines variables explicatives.

Tableau 1 : variables indépendantes

\begin{tabular}{llc}
\hline Variables & Modalités & Fréquence \% \\
\hline Age & $12-14$ ans & 14,1 \\
Sexe & $15-19$ ans (CR) & 85,9 \\
& Masculin & 43,7 \\
Milieu de socialisation & Féminin (CR) & 56,3 \\
& Bangui (CR) & 91,5 \\
Religion & Autres milieux & 8,5 \\
& Catholique (CR) & 51,6 \\
Situation d'activité & Protestant & 44,9 \\
& Autres & 3,5 \\
Prise en charge & Élèves (CR) & 76,5 \\
& Autres & 23,5 \\
Nombre de partenaires sexuels & Seul & 11,1 \\
& Parents (CR) & 57,8 \\
Fréquentation des services & Autres & 31,1 \\
d'éducation sexuelle & 0 & 23,8 \\
Communication en matière de & 1 (CR) & 48,4 \\
sexualité avec les parents & $2+$ & 27,9 \\
Perception des risques & Oui (CR) & 39,0 \\
& Non & 61,0 \\
Nombre de cas & Non (CR) & 50,8 \\
\hline & Oui, librement & 34,0 \\
& Oui, moins ouvertement & 15,2 \\
& Oui & 13,5 \\
& Non (CR) & 86,5 \\
& & 341 \\
\hline
\end{tabular}

$C R=$ catégorie de référence 
Il convient de signaler que les 341 individus ont été sélectionnés en tenant compte des pratiques sexuelles des adolescents. Ici, il ne s'agit pas de procéder à un nouveau tirage des individus mais une sélection sur la base du critère susmentionné.

\section{Résultats}

\section{Les facteurs affectant la sexualité des adolescents}

\section{Evaluation des conséquences des rapports sexuels précoces}

Dans un certain nombre de cas, le comportement d'un individu en matière de santé dépend de l'évaluation de ces conséquences. En effet, d'après les résultats de l'enquête, $88 \%$ des adolescents des deux sexes confondus connaissent les conséquences des rapports sexuels précoces. Mais curieusement ils se contredisent par rapport à leur comportement sexuel dans la mesure où $48 \%$ des adolescents avaient eu leur rapport sexuel avant 15 ans. Ils ont été nombreux à citer les grossesses non désirées, les maladies sexuellement transmissibles/Sida $(66,7 \%)$, suivi du Sida et de grossesse représentant respectivement $12,1 \%$ et 9,2 \%. Cela pourrait traduire en partie l'impact de différentes actions menées en faveur des jeunes. Il s'agit du Projet «Education à la Vie Familiale en Matière de Population (EVF/EMP), les actions de sensibilisation du Centre d'Information sur la Sexualité des Jeunes (CISJEU), et des clubs de jeunes pour la lutte contre le Sida dans les différents établissements scolaires de Bangui. Cependant, bien qu'ayant une connaissance plus ou moins exacte des conséquences de la sexualité précoce, près de la moitié des adolescents (48 \%) ont tout de même reconnu avoir leur premier rapport sexuel avant 15 ans. Ce qui suppose que l'acquisition des informations sur les conséquences de la sexualité précoce est postérieur à l'acte ou alors qu'elle n'a pas été suffisante pour atténuer le désir chez les adolescents d'expérimenter le rapport sexuel. Dans le premier cas, il peut y avoir une prise de conscience réelle du danger encouru se traduisant par l'adoption d'un comportement de prévention des risques (fréquences irrégulières, protection des rapports à l'avenir). Dans le second cas, où il ne s'agit plus d'une ignorance mais plutôt d'une incrédulité coupable, les prédispositions à un comportement de prévention des risques sont d'un faible degré. D'où la nécessité de rendre l'information disponible et accessible dès le bas âge pour faciliter une évaluation des conséquences pouvant influer effectivement sur la décision de passer à l'acte sexuel. Audelà de la connaissance des conséquences des rapports non protégés, des différents modes de transmission de ces infections, ainsi que les manifestations ou symptômes ne sont pas toujours connus.

\section{Perception des risques}

La perception des risques peut susciter l'adoption d'un comportement préventif en matière de sexualité, en l'absence des facteurs collatéraux puissants concomitants. Il y a la maîtrise de soi lorsqu'on se trouve en face 
d'un(e) partenaire sexuel(le). Parfois, la réaction du partenaire sexuel peut dissiper la bonne intention à adopter le comportement qu'on souhaitait adopter. D'après le résultat de l'enquête, 54,4 \% ont peur de mourir du Sida, contre $24 \%$ qui ont l'appréciation des trois conséquences des rapports non protégés à savoir : les grossesses non désirées, les MST et le SIDA. La peur de la grossesse ne réunit que $10 \%$ seulement. Si l'on s'en tient à l'opinion exprimée par les adolescents interrogés sur ce qui leur fait plus peur par rapport aux conséquences des rapports sexuels précoces, on serait tenté de penser qu'ils seraient plus enclins à se protéger systématiquement lors de tout rapport sexuel. Il convient de signaler que les filles ont davantage peur de la stérilité et des grossesses non désirées et trois conséquences à la fois par rapport aux garçons, avec des scores respectifs de $80,3 \% ; 67,7 \%$ et 59,5 $\%$. Par contre, les garçons dans $53,4 \%$ des cas déclarent la peur du SIDA comme risque encouru pendant l'acte sexuel.

Tableau 2 : Répartition des adolescents selon la perception de risque et sexe

\begin{tabular}{lrrrrrr}
\hline \multirow{2}{*}{$\begin{array}{l}\text { Perception des } \\
\text { risques }\end{array}$} & \multicolumn{4}{c}{ Sexe } & \multicolumn{3}{c}{ Ensemble } \\
\cline { 2 - 7 } & Masculin & \% & Féminin & Effectifs & \% \\
\hline Grossesse/MST/SIDA & 68 & 17,71 & 100 & 23,70 & 168 & 20,84 \\
VIH/SIDA & 205 & 53,39 & 179 & 42,42 & 384 & 47,64 \\
Grossesse & 20 & 5,21 & 42 & 9,95 & 62 & 7,69 \\
Stérilité & 13 & 3,39 & 53 & 12,56 & 66 & 8,19 \\
Autres & 15 & 3,90 & 9 & 2,13 & 24 & 2,98 \\
Sans opinion & 63 & 16,40 & 39 & 9,24 & 102 & 12,66 \\
Total & 384 & 100 & 422 & 100 & 806 & 100 \\
\hline
\end{tabular}

Cependant, parmi les moyens de protection contre les IST/SIDA énumérés par les adolescents figurent par ordre d'importance la fidélité $(48,8 \%)$, l'abstinence $(19,4 \%)$, la limitation du nombre de partenaires $(14,1 \%)$, le bon choix d'un partenaire $(10,2 \%)$ et l'utilisation des préservatifs $(7 \%)$. Il apparait donc que l'utilisation des préservatifs est citée en dernier lieu. Ce qui signifie qu'elle n'est pas considérée comme une pratique préférentielle par les adolescents qui privilégient pour leur part la fidélité. Or, la fidélité suppose une confiance totale et réciproque entre partenaires engagés dans une union durable. Dans le contexte des relations amoureuses entre adolescents généralement marquées par leur caractère occasionnel et éphémère, il est peu probable d'admettre l'applicabilité effective de ce principe dans la réalité. Dans ce cas précis, la distorsion entre la perception du risque et l'adoption d'un comportement préventif est très remarquable.

\section{Fréquentation des structures de santé sexuelle}

En matière de changement de comportement, le processus qui va de la prise de conscience à l'intention et de l'intention au comportement souhaité peut être facilité par un certain nombre de facteurs dont la présence des amis, l'existence des structures de santé sexuelle des adolescents, la pression des parents. Tout ceci constitue les éléments pouvant donner à l'adolescent 
l'habileté à agir selon la norme. Il ressort des données d'enquête que $55 \%$ des adolescents connaissent les lieux d'expressions des problèmes intimes ; $45 \%$ ignorent leur existence. Parmi ceux-ci qui connaissent ces lieux, $65 \%$ fréquentent les différents services de santé sexuelle des jeunes. Il convient de noter que presque la quasi-totalité de ceux qui fréquentent estiment que les services sont adaptés à leurs besoins.

\section{Pourquoi certains adolescents ne fréquentent-ils pas les structures d'encadrement des jeunes?}

Plusieurs raisons ont été évoquées pour justifier la non-fréquentation des services de santé des jeunes. $24 \%$ affirment qu'ils n'ont pas le temps, suivis de ceux qui évoquent le manque d'habitude de fréquenter ces lieux (9,5\%) et de ceux qui ne le font pas à cause de l'éloignement de leur domicile $(8,7 \%)$. D'autres enfin pensent qu'ils sont encore trop jeunes; ils restent sous surveillance des parents, par conséquent, il ne peuvent pas se promener sans l'avis de leurs parents : 9,2 \% des adolescents l'ont affirmé. Si les éducateurs de santé sexuelle des jeunes ne peuvent atteindre facilement les plus jeunes qui sont souvent contraints de se soumettre à la volonté de leurs parents, leurs parents pourraient valablement jouer ce rôle dans un cadre familial si ces problèmes ne se discutaient pas à mots couverts avec les enfants.

\section{Communication parents-adolescents}

La communication est un processus interactif de dialogue entre deux individus (ici parents-adolescents) dont le but ultime est de persuader un individu à adopter un comportement sain. Les données d'enquête montrent que seulement $42,6 \%$ des enfants parlent de la sexualité avec leurs parents, parmi lesquels $60,6 \%$ parlent assez librement et $39,4 \%$ à mots couverts. Ceci traduit la volonté des parents de considérer la sexualité comme un sujet tabou. Même si l'on parle, on se contente d'attirer l'attention de l'enfant sur les conséquences des rapports précoces ou des rapports protégés. Mais on ignore qu'à l'âge de la maturité, il y a un certain nombre de réactions naturelles que l'adolescent doit avoir en fonction de son âge. Dans la communication, les parents ont tendance à évoquer les conséquences des rapports sexuels sans pour autant montrer à l'enfant comment utiliser son sexe pendant l'âge de puberté. Dès lors que l'adolescent n'est pas convaincu pour adhérer au point de vue des parents, il est souvent difficile d'opérer un changement à ce niveau.

Les parents doivent s'investir davantage dans l'éducation sexuelle de leurs enfants eu égard à la confiance que les adolescents leur font. En effet, concernant la question "qui doit vous fournir les informations sur la sexualité ? ", il ressort que 36,4 \% des adolescents pensent que les parents sont habilités à le faire quand bien même ces derniers ont des limites. Le personnel de santé, les personnes âgées viennent loin derrière avec respectivement $27,8 \%$ et $11,9 \%$. Les filles préfèrent avoir comme interlocuteurs en matière de sexualité, leurs parents et enseignants avec 
respectivement $62,8 \%$ et $74,5 \%$. En revanche, les garçons ont une préférence pour les personnes âgées, les religieux, les amis et le personnel de santé avec respectivement $70,8 \%, 65,5 \%, 53,1 \%$ et 52,7. D'ailleurs, il ressort de l'analyse du contenu des discussions de groupe avec les adolescents que ces derniers, quel que soit leur sexe, ont mis en évidence le déficit de communication entre parents et enfants en matière de sexualité. Un déficit qui se caractérise soit par une insuffisance d'information au niveau familial, soit par un empêchement des enfants à avoir accès à d'autres sources d'information disponibles dans la ville. Les filles ont affirmé que «certains parents empêchent leurs enfants (filles) de fréquenter le Centre d'Information sur la Sexualité de Jeunes (CISJEU) sous prétexte qu'elles répondent à des rendez-vous des garçons ». Quant aux garçons, ils ont reconnu que "généralement, c'est après le constat d'un problème de sexualité (grossesse ou défloration de mineure) que les parents commencent à éduquer les enfants ». Ces données confirment ainsi la tendance généralement admise selon laquelle dans les sociétés, la sexualité est considérée comme un sujet tabou par les parents qui sont en fait les premiers éducateurs des enfants.

\section{Analyse bivariée : description du comportement de prévention}

Le comportement de prévention est généralement caractérisé par le retardement de l'activité sexuelle jusqu'à la maturité physiologique et la protection des rapports sexuels. Sur la protection des rapports sexuels, les opinions des adolescents recueillies au cours des discussions de groupes sont caractérisées par une certaine ambivalence. Les adolescents ont reconnu que certains d'entre eux qui sont consciencieux et soucieux de leur avenir utilisent le préservatif au cours des rapports sexuels alors que d'autres ne l'utilisent pas. Aussi l'attitude vis-à-vis du port du préservatif est-elle caractérisée soit par une intransigeance soit par une indifférence. En général, l'exigence du port du préservatif par l'un des partenaires est souvent perçue par l'autre comme un manque de confiance de la part du (de la) partenaire d'où la nécessité d'une discussion préalable à l'instauration d'un climat d'entente et de bonne compréhension indispensable pour la continuité des relations amoureuses. Par ailleurs, les garçons ont affirmé qu' " au cours des relations sexuelles, certaines filles demeurent indifférentes et laissent le choix de la décision de protection à leurs partenaires ». L'attitude d'indifférence traduit une hésitation entre la nécessité de se protéger contre les conséquences de l'acte et le désir de vivre l'intense plaisir (extase) souvent associé au contact direct d'une relation sexuelle sans capote) : « d'autres refusent le port du préservatif parce qu'il empêche de sentir le vif plaisir du contact direct » ont déclaré les adolescents.

A titre de rappel, selon la théorie de l'action raisonnée, l'intention d'une personne d'adopter ou de ne pas adopter un comportement est déterminée par son attitude à l'égard du comportement et l'importance quelle accorde à l'opinion des gens qui lui sont proches. Le modèle des croyances relatives à la santé pose comme postulat qu'un individu est susceptible de poser un 
acte pour prévenir une maladie ou une condition désagréable s'il possède des connaissances minimales en matière de santé et s'il considère la santé comme une dimension d'importance dans sa vie. Les déterminants de la décision d'agir sont la perception d'une menace pour la santé et la croyance en l'efficacité de la menace pour la santé ainsi que la croyance en l'efficacité de l'action devant la menace provient de l'évaluation des avantages et des désavantages associés à l'adoption des actions préventives recommandées. De plus, certaines variables (démographiques, socio psychologiques) influent sur les perceptions de l'individu et certains évènements incitant à l'action peuvent éveiller chez lui la perception d'une menace pour sa santé.

\section{Variables d'identification sociale}

Le comportement sexuel est fonction de l'âge. En effet, la pratique de l'acte sexuel est déterminée par la maturité d'un individu. Au fur et à mesure que l'âge augmente, la proportion des adolescents ayant déclaré avoir eu des rapports sexuels augmente. Cette tendance est similaire au comportement de prévention où l'utilisation des préservatifs au cours du dernier rapport sexuel évolue avec l'âge de l'adolescent. Aussi, il apparaît, d'après le tableau ci-dessus, que c'est chez les adolescents de 18 à 19 ans qu'on retrouve les scores élevés de ceux qui ne se protègent pas lors du dernier rapport sexuel. Il existe une liaison entre l'âge et le comportement de prévention chez les adolescents. Ce comportement qui est de plus en plus répandu en milieu scolaire doit aussi interpeller les parents des adolescents.

La variable sexe permet d'expliquer la différence de comportement sexuel $\mathrm{du}$ point de vue de la prévention. Au seuil de $10 \%$, cette variable s'est révélée significative, d'après les résultats de l'enquête, $60 \%$ des garçons se sont protégés lors de leur dernier rapport sexuel contre $40 \%$ de filles. Il va falloir nuancer ce résultat dans la mesure où chez les filles, la latitude est souvent laissée au garçon de décider de porter le préservatif ou pas. Ceci dit, il ne s'agit pas ici de l'utilisation du préservatif féminin qui vient d'être vulgarisé très récemment en Centrafrique. 
88 African Population Studies Vol.20 $n^{\circ}$ 2/Etude de la population africaine vol. $20 n^{\circ} 2$

Tableau 3 : Analyse bivariée des variables indépendantes et le comportement de Prévention

\begin{tabular}{|c|c|c|c|}
\hline \multirow[t]{2}{*}{ Variables Indépendantes } & \multicolumn{3}{|c|}{$\begin{array}{l}\text { Variables de Comportement de } \\
\text { Prévention }\end{array}$} \\
\hline & Oui & Non & Signification \\
\hline Age & & & $0,001(\mathrm{~s})$ \\
\hline $12-14$ & $5,6 \%$ & $22,5 \%$ & \\
\hline $15-17$ & $45,8 \%$ & $34,3 \%$ & \\
\hline $18-19$ & $48,2 \%$ & $39,1 \%$ & \\
\hline Sexe & & & 0,089 \\
\hline Masculin & $59,7 \%$ & 50,5 & \\
\hline Féminin & $40,3 \%$ & $49,5 \%$ & \\
\hline Connaissances des conséquences $d$ & & & \\
\hline précoces & & & 0,220 \\
\hline Oui & 89,6 & 86,2 & \\
\hline Non & $10,4 \%$ & 13,8 & \\
\hline Laquelle fait peur & & & $0,028 \quad(\mathrm{~s})$ \\
\hline Grossesse/MST/Sida & 31,0 & 25,4 & \\
\hline Sida & 45,02 & 54,4 & \\
\hline Grossesse & 7,8 & 5,6 & \\
\hline Stérilité & 10,9 & 10,7 & \\
\hline Croissance/social/Psychologique & 5,4 & 2,9 & \\
\hline Qui fait changer d'avis & & & 0,452 (ns) \\
\hline Parents/Famille & $27,3 \%$ & $30,1 \%$ & \\
\hline Amis & $4,9 \%$ & 3,0 & \\
\hline Frères / Sœur & $4,9 \%$ & 2,0 & \\
\hline Personne & $62,9 \%$ & $65 \%$ & \\
\hline Communication parent & & & $0,000 \quad(\mathrm{~s})$ \\
\hline Oui & 67,2 & 29,0 & \\
\hline Non & 32,8 & 70,1 & \\
\hline Fréquentation Lieux/Service & & & $0,000(\mathrm{~s})$ \\
\hline Oui & 77,8 & 54,0 & \\
\hline Non & 22,2 & 46,0 & \\
\hline Qui fournit les informations & & & 0,519 (ns) \\
\hline Parents & 26,2 & 32,0 & \\
\hline Ami(e) & 7,1 & 6 & \\
\hline Personnel de Santé & 36,9 & 35,0 & \\
\hline Enseignant & 7,1 & 3,5 & \\
\hline Personnes âgées & 10,6 & 13,0 & \\
\hline Pasteur & 3,5 & 5,0 & \\
\hline Autres & 8,5 & 5,5 & \\
\hline
\end{tabular}

$S=$ significatif

Ns = non significatif 


\section{Variables intermédiaires}

Il apparaît d'après le tableau ci-après qu'il n'y a aucune liaison entre l'évaluation des conséquences et les actes concrets liés à la prévention; au seuil de $10 \%$ elle est non significative. Il n'y pas une indifférence de comportement sexuel en fonction de la connaissance des conséquences. D'après la théorie de Azjen et Fishbein, 1980, l'attitude d'une personne à l'égard du comportement est déterminée par ses croyances concernant les conséquences d'un comportement donnée et l'évaluation qu'elle fait de ces conséquences. Il s'agit en fait du jugement subjectif par rapport à un comportement et à ses conséquences ou résultats possibles multiplié par l'évaluation que fait la personne de ces conséquences. D'une manière plus concrète, on ne peut pas dire que l'adolescent associe des conséquences aux actions qu'il pose (croyances) et qu'il attribue une valeur à ces conséquences.

Bien qu'il y ait une liaison entre la perception $d u$ risque des rapports sexuels non protégés et le comportement de prévention, le Sida constitue pour les adolescents la conséquence principale qui leur fait le plus peur : plus de la moitié des adolescents (56\%) qui ont un comportement à haut risque ont curieusement cité le Sida, contre un quart $(25 \%)$ qui redoutent les deux autres conséquences à savoir les grossesses non désirées et les maladies sexuellement transmises.

Il ressort des résultats de l'enquête que la croyance à l'opinion des personnes influentes n'a aucune liaison avec la variable de comportement de prévention. Ainsi, quel que soit leur comportement sexuel, plus de la moitié des adolescents ne reçoit aucune pression quelconque dans le choix de leur partenaire sexuel. Il convient de noter qu'un peu plus d'un quart des adolescents ont opté pour les parents, quand il s'agit de changer d'opinion dans la décision de choisir un partenaire sexuel. Ce résultat traduit la faible emprise des parents sur leurs enfants en matière de prise de décision.

Cependant, la communication apparaît, d'après le test statistique, comme une variable explicative du comportement de prévention. Il ressort du tableau d'analyse que les adolescents qui communiquent avec leurs parents sont les plus nombreux à avoir un comportement à moindre risque. En revanche, ceux qui ont un comportement à haut risque ont déclaré ne pas communiquer avec leurs parents. Pour que les actions entreprises avec les ONGs ou les associations oeuvrant en direction des jeunes puissent porter des fruits, il va falloir que les personnes en charge de l'éducation des enfants puissent tenir le même langage. Car, lorsque les parents ont une faible emprise sur les enfants qui se traduit par l'absence de communication, il est difficile d'atteindre le but recherché qui est d'avoir un comportement sexuel à moindre risque. Ceci nous amène à aborder la question des facteurs qui facilitent ou renforcent l'action.

Les facteurs de renforcement du comportement de prévention des adolescents sont entre autres la tendance des structures d'encadrement des jeunes 
en matière de sexualité. Il ressort des données d'enquête que les adolescents qui connaissent les lieux d'expression des problèmes intimes ont tendance à adopter un comportement sexuel à moindre risque par rapport à ceux qui n'ont pas connaissance de ces conséquences des rapports sexuels non protégés. C'est ainsi que le fait de connaître ces structures ne fait que renforcer le comportement sexuel souhaité (abstinence ou port de préservatif). Un fait tout à fait curieux est la prédominance de ceux qui sont informés au niveau des centres d'éducation sexuelle sur ceux qui ne s'intéressent pas aux structures d'encadrement des jeunes. Cela traduit en partie l'échec des interventions éducatives lorsque l'environnement immédiat de la population cible n'est pas pris en compte dans la stratégie d'action. L'étape qui va de la connaissance à l'intention d'agir dans le sens souhaité est souvent longue et difficile à planifier. La même tendance est observée au niveau de la fréquentation des structures d'encadrement des jeunes où la fréquentation des structures a une forte liaison avec le comportement de prévention. Plus de trois quart des adolescents qui fréquentent les structures ont des prédispositions à protéger leurs rapports sexuels.

Il apparaît, d'après les résultats de l'enquête, que quel que soit le comportement sexuel adopté par l'adolescent, le personnel médical vient en tête avec plus d'un tiers, suivi des parents qui, de temps en temps, complètent les informations données par le personnel médical. Ceci pourrait s'expliquer par les actions entreprises par les autorités sanitaires en vue de réduire l'ampleur des maladies sexuellement transmissibles et le Sida parmi les jeunes : des structures d'éducation et de dépistage ont été créées pour la prise en charge des problèmes des jeunes en matière de sexualité. Il convient de noter que la variable "personne ressource pour l'obtention des informations sur la sexualité » n'a aucune liaison avec le comportement de prévention. $C^{\prime}$ est dire que quelle que soit la source d'information sur la sexualité, le comportement sexuel des adolescents de Bangui est identique.

\section{Analyse multivariée de l'utilisation du préservatif}

\section{Les effets des variables individuelles}

Les caractéristiques individuelles des adolescents sont appréhendées à travers l'âge, le sexe, le niveau d'instruction, la situation d'activité, la religion, le milieu de socialisation et la prise en charge de l'adolescent.

Il ressort du tableau ${ }^{\circ} 4$ que certaines variables ont un impact significatif sur la décision d'utilisation du préservatif lors des rapports sexuels.

D'abord, on s'aperçoit que la variable âge exerce une influence significative sur l'utilisation de préservatif pendant les rapports sexuels chez les adolescents. Les adolescents du groupe d'âge 15-19 ans ont au niveau brut 5 fois plus de chance d'utiliser le préservatif que ceux âgés de 12-14 ans. Cette influence de l'âge sur les comportements sexuels des adolescents demeure significative lorsqu' on introduit la variable relative dans le modèle. Comme 
on peut le constater dans le modèle 2 , lorsqu'on introduit la variable relative à la perception des risques liés aux rapports sexuels non protégés ainsi que le nombre des partenaires sexuels, il ressort que les chances de port de préservatifs sont 4,7 fois plus élevées chez les adolescents du groupe d'âges 15-19 ans que ceux de 12-14 ans. Il en est de même lorsqu'on prend en compte l'effet des variables sur la fréquentation des services d'éducation sexuelle et sur la communication avec les parents en matière de sexualité. Autrement dit, une partie de l'influence de l'âge sur l'utilisation du préservatif est médiatisée par la différence du nombre de partenaires sexuels, de fréquentation des services d'éducation sexuelle et de communication avec les parents. Dans ces domaines, les plus jeunes seraient défavorisés. Si l'effet de la variable sexe apparaît non significatif dans les modèles 1 et 2, il ressort toutefois que les garçons ont tendance à plus utiliser le préservatif lors de leurs rapports sexuels que les filles. Lorsqu'on contrôle les effets de la fréquentation des services d'éducation sexuelle et la communication avec les parents en matière de sexualité, l'effet de la variable sexe devient significatif. Les garçons présentent $62 \%$ de chances de plus d'utiliser de préservatif lors de leurs rapports sexuels de l'analyse que les filles.

Pour ce qui est du type d'activité, les résultats de l'analyse de la régression logistique indiquent que cette variable ne présente aucun effet significatif sur les comportements sexuels en matière d'utilisation du préservatif chez les adolescents. Mais si l'on compare les chances d'utilisation de préservatif entre adolescents qui fréquentent un établissement scolaire aux autres en tenant compte des effets des autres variables, on se rend compte que les chances d'utilisation de préservatif sont plutôt de 32 \% plus élevées chez les élèves que chez les autres adolescents, toutes choses égales par ailleurs. Aussi, les effets d'utilisation des préservatifs lors du dernier rapport sexuel des autres variables relatives aux caractéristiques individuelles telles que la prise en charge des adolescents, la religion et le milieu de socialisation se sont avérés significatifs.

\section{Les effets des comportements sexuels}

Les comportements sexuels des adolescents sont appréhendés par deux variables : la perception des risques liés aux relations sexuelles non protégés et le nombre de partenaires sexuels au cours de trois derniers mois précédant l'enquête. Ces deux variables ont été introduites après avoir pris en compte les variables relatives aux caractéristiques individuelles (modèle 2). Il y a lieu de remarquer que l'introduction de ces deux variables n'a pas changé significativement les effets des autres variables. La perception des risques liée à la sexualité non protégée n'exerce pas une influence significative sur l'utilisation du condom par les adolescents. Mais on note une différence assez importante entre les adolescents qui sont conscients des risques liés aux rapports sexuels non protégés et à ceux qui les ignorent. En effet, les adolescents qui connaissent les risques des rapports sexuels non protégés auraient deux fois plus de chance d'utiliser un préservatif lors de leurs rapports sexuels que ceux qui en ignorent les dangers. Cependant, on 
remarque que ce risque relatif diminue lorsqu' on introduit des variables sur la communication en matière de sexualité. En ce qui concerne le nombre de partenaires sexuels, son influence sur les chances d'utilisation de préservatif se révèle significative au seuil de $5 \%$. Par contre, le fait de n'avoir qu' un seul partenaire n'influence pas le port de préservatif pendant les rapports sexuels. Les adolescents ayant déclaré avoir plus de deux partenaires sexuels ont $71 \%$ de chance de plus d'utiliser le préservatif que ceux qui ont un seul partenaire. En d'autres termes, plus le nombre de partenaires est élevé, plus grande est la chance que l'adolescent utilise un préservatif lors du rapport sexuel.

\section{L'impact de la communication en matière de sexualité}

La communication en matière de sexualité est mesurée à travers deux principales variables : la fréquentation des services d'éducation sexuelle et la communication avec les parents en matière de sexualité. L'introduction de ces variables dans le modèle induit des effets significatifs sur le comportement des adolescents en matière de port de préservatif. Les adolescents qui ne fréquentent pas les services d'éducation sexuelle courent un risque de ne pas utiliser le préservatif lors des rapports sexuels de $36 \%$ supérieur à celui de ceux qui le font.

La communication en matière de sexualité avec les parents se révèle également un facteur important de différenciation. L'effet de cette variable sur l'utilisation de préservatif est significatif. Cependant, il y a lieu de noter que cet effet varie en fonction du degré d'ouverture d'esprit des parents en matière de communication et de sensibilisation sur la sexualité de leurs enfants. En fait, les adolescents qui parlent moins ouvertement de sexualité avec leurs parents, présentent seulement deux fois plus de chances d'utilisation du préservatif que ceux qui ne bénéficient pas d'une telle communication avec leurs parents. Par contre, les adolescents qui parlent librement de sexualité avec leurs parents sont trois fois plus enclins à utiliser le préservatif que ceux qui n'en parlent pas. 
Tableau 4 : Déterminants de l'utilisation de préservatifs lors des rapports sexuels des adolescents (12-19 ans) ayant eu au moins un rapport sexuel et résidant à Bangui (coefficient et risques relatifs de la régression logistique)

\begin{tabular}{|c|c|c|c|c|c|c|}
\hline \multirow[t]{2}{*}{ Variables } & \multicolumn{2}{|c|}{ Modèle 1} & \multicolumn{2}{|c|}{ Modèle 2} & \multicolumn{2}{|c|}{ Modèle 3} \\
\hline & Coef. & $\begin{array}{l}\text { Risque } \\
\text { relatif }\end{array}$ & Coef. & $\begin{array}{l}\text { Risque } \\
\text { relatif }\end{array}$ & Coef. & $\begin{array}{l}\text { Risque } \\
\text { relatif }\end{array}$ \\
\hline \multicolumn{7}{|l|}{ Groupe d'âge } \\
\hline $12-14$ ans & $-1,666^{* *}$ & 0,188 & $-1,537^{* *}$ & 0,215 & $-1,423^{* *}$ & 0,240 \\
\hline 15-19ans (CR) & - & 1,000 & - & 1,000 & - & 1,000 \\
\hline \multicolumn{7}{|l|}{ Sexe } \\
\hline Féminin (CR) & - & 1,000 & - & 1,000 & - & 1,000 \\
\hline Masculin & 0,394 & 1,483 & 0,294 & 1,341 & $0,482^{*}$ & 1,620 \\
\hline \multicolumn{7}{|l|}{ Situation d'activité } \\
\hline Élève (CR) & - & 1,000 & - & 1,000 & - & 1,000 \\
\hline Autres & $-0,419$ & 0,657 &,- 476 & 0,621 & $-0,386$ & 0,679 \\
\hline \multicolumn{7}{|l|}{ Prise en charge } \\
\hline Parents (CR) & - & 1,000 & - & 1,000 & - & 1,000 \\
\hline Autres personnes & $-0,312$ & 0,731 & $-0,323$ & 0,724 & $-0,239$ & 0,787 \\
\hline Seul & 0,071 & 1,074 & 0,115 & 1,122 & 0,294 & 1,343 \\
\hline \multicolumn{7}{|l|}{ Religion } \\
\hline Catholique (CR) & - & 1,000 & - & 1,000 & - & 1,000 \\
\hline Protestant & 0,116 & 1,123 & 0,151 & 1,163 & 0,191 & 1,210 \\
\hline Autres & 0,202 & 1,224 & 0,233 & 1,263 & 0,336 & 1,399 \\
\hline \multicolumn{7}{|l|}{ Milieu de socialisation } \\
\hline Bangui (CR) & - & 1,000 & - & 1,000 & - & 1,000 \\
\hline Provinces & $-0,091$ & 0,912 & $-0,059$ & 0,942 & 0,001 & 1,001 \\
\hline \multicolumn{7}{|l|}{$\begin{array}{l}\text { Perception des risques liés aux } \\
\text { relations sexuelles }\end{array}$} \\
\hline Oui & & & 0,681 & 1,976 & 0,395 & 1,485 \\
\hline Non $(C R$ & & & - & 1,000 & - & 1,000 \\
\hline \multicolumn{7}{|l|}{ Nombre de partenaires sexuels } \\
\hline 0 & & & $-0,042$ & 1,043 & $-0,109$ & 1,116 \\
\hline $1(\mathrm{CR})$ & & & - & 1,000 & - & 1,000 \\
\hline $2 \mathrm{ou}+$ & & & $0,759^{*}$ & 1,582 & $0,728^{*}$ & 1,712 \\
\hline \multicolumn{7}{|l|}{ d'éducation sexuelle } \\
\hline Oui (CR) & & & & & - & 1,000 \\
\hline Non & & & & & $-0,743^{* *}$ & 0,642 \\
\hline \multicolumn{7}{|l|}{ Communication avec les parents } \\
\hline Non $(C R)$ & & & & & - & 1,000 \\
\hline Moins ouvertement & & & & & $0,816^{* *}$ & 2,262 \\
\hline Librement & & & & & $1,0981^{* *}$ & 2,998 \\
\hline Nombre de cas & & & & & & \\
\hline Log de vraisemblance & & 15 & & & 415 & \\
\hline $\mathrm{R}^{2}$ (Nagelkerke) & & & & & & \\
\hline
\end{tabular}

** $\quad$ significatif au seuil de 1\%

* $\quad$ significatif au seuil de 5\%

(CR) Catégorie de référence 


\section{L'incidence de l'ensemble des variables sur le comportement de la prévention}

Il convient de rappeler que, la plupart des adolescents interrogés sont des célibataires $(95 \%)$. Leurs rapports sexuels devraient être protégés pour éviter les conséquences néfastes qui pourraient contribuer à compromettre leur avenir. Etant donné que le niveau de conscience des perceptions de risque ne suit pas le comportement sain qui devait être observé par les adolescents, quels sont les différents facteurs susceptibles d'agir sur la décision à utiliser le préservatif pour protéger les rapports sexuels ?

Lorsqu' on considère globalement l'incidence de l'ensemble des variables sur le comportement préventifs le fait de fréquenter l'école, ou d'être socialisé à Bangui ou à l'intérieur du pays ne fait pas varier le comportement sexuel des adolescents. Par contre, la fréquentation des structures de santé des jeunes, la communication avec les parents, l'âge, le nombre de partenaires sexuels depuis le premier rapport sexuel se sont révélés significatif et expliquent donc la variation du comportement sexuel sain.

La présence des structures d'éducation sexuelles est un des facteurs renforçant ou facilitant le comportement de prévention des adolescents. Bien que la communication joue un rôle de déterminant dans le processus du maintien du comportement sexuel des adolescents, en matière de sexualité, la décision d'adopter un comportement dépend également de l'attitude du ou (de la) partenaire chez laquelle, il faut une conscience assez forte des perceptions du risque avant d'imposer sa volonté à son ou (sa) partenaire sexuel(le).

Dans le troisième modèle, les variables non significatives ont été extraites et ne figurent dans le modèle que les variables qui ont un pouvoir prédictif élevé par rapport à la variable expliquée. Ici, le $\mathrm{R}^{2}$ connaît une légère baisse (14\%). En définitive, le comportement sexuel des adolescents est déterminé par certaines variables d'identification sociale telles que : l'âge, le sexe et les variables contextuelles telles que la communication avec les parents, la fréquentation des structures d'encadrement des jeunes etc.

\section{Conclusion}

Il ressort de nos analyses que les résultats vont de manière générale dans le sens des hypothèses énoncées.

En ce qui concerne l'hypothèse selon laquelle les adolescents appartenant au groupe d'âges jeunes et dont le milieu de socialisation est autre que Bangui sont plus enclins aux rapports sexuels non protégés, au regard des résultats obtenus, il ressort évidemment que l'appartenance au groupe d'âge 12-14 ans est associée à une faible utilisation du préservatif lors des rapports sexuels. En revanche, le milieu de socialisation de l'enfant n'exerce aucun effet significatif sur les comportements en matière d'utilisation de préservation lors des rapports sexuels. 
Il convient également de souligner que la fréquentation des services d'éducation sexuelle, la communication en matière de sexualité avec les parents et le multipartenariat se sont avérés déterminants dans les comportements sexuels des adolescents résidant à Bangui. Ce résultat est presque conforme à l'une des hypothèses de cette étude qui soutient que l'utilisation par les adolescents des préservatifs pendant les rapports sexuels est fonction de la fréquentation des services d'éducation sexuelle, de communication avec leurs parents, du nombre de partenaires sexuels et de la perception des risques des rapports sexuels non protégés.

Enfin, s'il apparaît que les objectifs de cette étude sont de manière générale atteints, il y a cependant lieu de noter que les résultats de la régression logistique peuvent comporter certaines limites imputables en grande partie aux données utilisées. Par exemple, la méthode adoptée pour collecter les données de l'enquête pourrait induire le biais de sélection. A cela, il convient aussi de souligner la taille réduite de l'échantillon qui pourrait limiter l'efficacité des coefficients estimés. Nonobstant cela, on peut retenir de cette étude les principaux constats suivants :

1) On assiste à l'érosion des valeurs socioculturelles qui se traduit par l'affaiblissement du pouvoir des aînés sur les jeunes ; il s'ensuit que l'éducation des enfants préoccupe de moins en moins la communauté.

2) La prise de conscience de la contraception est plus faible dans la population adolescente au moment de l'enquête, alors que c'est dans ce groupe d'âge qu'on retrouve le pourcentage des personnes instruites le plus élevé.

3) Le comportement sexuel à risque peut contribuer à terme à l'augmentation de la mortalité générale dont les conséquences immédiates sont la perte de la potentialité de reproduction future.

Il conviendrait de souligner que si incomplète soit-elle, cette étude est une contribution importante à l'amélioration de la connaissance des déterminants des comportements sexuels des adolescents à Bangui. Toutefois, étant donné l'importance du sujet, d'autres investigations seraient nécessaires pour mieux cerner tous les paramètres dont la maîtrise permettrait la définition des stratégies globales de promotion de la sexualité responsable en Centrafrique. Afin d'assurer une protection efficace de la jeunesse contre les conséquences néfastes d'une sexualité mal maîtrisée, il serait par exemple intéressant de mener une recherche sur la nécessité d'une éventuelle vulgarisation de l'éducation sexuelle comme facteur favorisant l'adoption des comportements à moindre risque chez les adolescents de l'école primaire.

Aussi, des investigations sur les causes profondes du relâchement du contrôle social sur les enfants et plus particulièrement en ce qui concerne la 
sexualité, seraient utiles dans le cadre d'une stratégie visant une meilleure implication de la communauté et des parents dans le processus d'éducation sexuelle des adolescents. Enfin, il est question d'étudier l'environnement familial des adolescents en mettant un accent sur le temps que les parents consacrent à l'éducation de leur enfant.

\section{Références}

Akoto E. 1993. Déterminants socio-culturels de la mortalité des enfants en Afrique

Noire, Hypothèses et recherches d'explication. Institut Catholique de Louvain, Instituts de Démographie, Académicia.

Akuffo F.O. 1987. Teenage Pregnancies and School Dropouts: The Relance of Family Life Education and Vocational Training to Girls Employment Opportunities, in C.Oppong ed., Sex Roles, Population and Development in West Africa, Heinemann, Portsmouth, N.H, and James Currey, London, pp. 128-154,

AnNDENISE B. 2000. Sexual Relations among Young People in Developing Countries: Evidences from WHO Cases Studies, Geneva, WHO.

Bamikale Feyisetan and R.Pebley A. 1989. Premarital Sexuality in Urban Nigeria, Studies in Family Planning, Vol.20, n6 pp 343-354, Novembre-Décembre.

Banque Mondiale. 1998. Note sur la pauvreté en République Centrafricaine.

BeAt-Songue P. 1998. "Influence du milieu social sur la sexualité et les comportements reproducteurs des adolescents au Sud Cameroun ", in KUATEDEFO B. (dir.), Sexualité et santé reproductive durant l'adolescence en Afrique, Boucherville, Ediconseil Inc., p. 177-192.

Blankhart D. 1997. Evaluation des problèmes des jeunes femmes enceintes à Bangui,

Bond V. and Dover P. (1997), « Men, Women and Trouble with Condoms: Problems Associated with Condom Use by Migrant Workers in Rural Zambia », in Health Transition Review, Supplement to volume 7, p. 377-391.

Bozon M. et Leridon H. 1993. Sexualité et sciences sociales, INED-PUF, Paris, Septembre Octobre, Numéro 5.

Calvès A.E., Cornwell G.T. and Enyegue P. 1996. Adolescent Sexual Activity in SubSaharan Africa: Do Men Have the Same Strategies and Motivations as Women? University Park, Pennsylvania, Pennsylvania State University, Working paper, $34 \mathrm{p}$.

Calvès A.E. 1996. Youth and Fertility in Cameroon: Changing Patterns of Family Formation, The Pennsylvania State University, 254 p. (Thesis of Rural Sociology and Demography).

Calvès A.E. 1998. "La sexualité pré maritale des adolescents à Yaoundé », in KUATEDEFO B. (dir.), Sexualité et santé reproductive durant l'adolescence en Afrique., Boucherville, Ediconseil Inc., p. 15-26.

Cherlin À. and Riley N.E. 1986. Adolescent Fertility: an Emerging Issue in Sub-Saharan Africa, Washington D.C, The World Bank (PHN Technical note 86-23).

Desgrées Du Loû A. 1997. SIDA et santé de la reproduction en Afrique subsaharienne. Une revue commentée de la littérature, Paris, Notes et Projets, ETS, ORSTOM, 25 p.

Ceped. 1997. La sexualité des adolescents au Sahel in Chronique du CEPED, Avril-Juin $\mathrm{n}^{\circ} 25$.

Cerpod. 1998. Les jeunes en danger, résultats d'une étude régionale, Bamako.

Cherlin Andrew and Nancy Riley. 1986. Adolescent Fertility: An Emerging Issue in Sub-Saharan Africa Healthy and Nutrition Department, Washington D.C: World Bank, July. 
Diop Nafissatou J. 1995. La fécondité des adolescents au Sénégal: Rapport d'étude, Programme de Petites Subventions de Recherche en population et développement, Mars.

Eggleston E., Leitch J. and Jackson J. 2000. «Consistency of Self-Reports of Sexual Activity among Young Adolescents in Jamaica », in International Family Planning Perspectives, vol. 26, n² 2, p. 79-83.

Evina À. 1990. L'infécondité en Afrique subsaharienne, Yaoundé, IFORD, Cahiers de l'IFORD n $1,281 \mathrm{p}$.

Evina A. 1998. Vie féconde des adolescentes en milieu urbain camerounais, cahier de l'IFORD

$\mathrm{n}^{\circ} 16$, Janvier.

Gage B. and Meekers D. 1992. "Conception and Childbearing before Marriage in Subsaharan Africa ", in International Family Planning Perspectives, vol. 19, $\mathrm{n}^{\circ} 1$, p. 14-18.

Gage B. and Meekers D. 1992. "Sexual Activity before Marriage in Subsaharan Africa ", Pennsylvania States University, Population Issues Research Center, $24 \mathrm{p}$.

Gérard Hubert. 1989. Théories et théorisation, in Josiane DUCHENE \& Guillaume WUNSCH éds., L'explication en sciences sociales. La recherche des causes en démographie, Chaire Quetelet'87, Louvain-la-Neuve, CIACO, pp. 267-281.

Gueye M., Castle S. and Kani-Konate M. 2001. «Timing of First Intercourse among Malian Adolescents: Implications for Contraceptive Use », in International Family Planning Perspectives, vol. 27, $\mathrm{n}^{\circ}$ 2, p. 56-62.

Gradwige M. 1986. Méthodes de recherche en Sciences Sociales, Dalloz, Paris.

Gobato I. 1996. Étude des comportements de recherche de soins des adolescents vivants à Bangui (RCA), Rapport final, PAJERS, GTZ, Juin.

Görgen ® et Katzan J. 1995. La sexualité des jeunes, résultats d'une étude qualitative sur les attitudes, les croyances et comportements des jeunes de Bangui, Tome 1: Analyse des données, Instituts de la médecine tropicale et de la Santé Publique RFA, Septembre.

Ilinigumugabo A. et al. 1996. "Causes and conséquences of adolescent pregnancy in Cameroon », Center for African Families Studies, 98 p.

Ibrahima M. 1999. Les déterminants de la sexualité, de la nuptialité et de la maternité précoces chez les adolescentes au Niger, Yaoundé, IFORD, 126 p. (Mémoire de DESS en démographie).

Juarez F. 1998. Do Reproductive Health Risks Vary among Low Incomes Youths? Dublin, British Society of Population Studies.

Kadjouna D. 1997. Enquête CAP auprès des tenanciers et filles libres, KM36 Mamou et Tamagaly, Ministère de la Santé Publique de la République de Guinée N59/60 Décembre 96 Janvier.

Kaptue L. 1998. "Les adolescents et le VIH/SIDA: le cas du Cameroun », in KUATEDEFO B. (dir), Sexualité et santé reproductive durant l'adolescence en Afrique, Boucherville, Ediconseil Inc., p. 287-296.

Kone H. SY J.H. 1995. La communication pour le développement durable, PUCI.

Kouton E. 1992. Évaluation et recherche des facteurs de la fécondité précoce au Bénin,

Cahiers de l'IFORD, Décembre.

Kuate-Defo B. 1998a. "Tendances et déterminants de l'activité sexuelle à l'adolescence ", in KuATE-DEFO B. (dir), Sexualité et santé reproductive durant l'adolescence en Afrique, Boucherville, Ediconseil Inc., p. 63-80.

Kuate-Defo B. 1998b. "Tendances et determinants des variations régionales de l'activité sexuelle prémaritale à l'adolesence », in KUATE-DEFO B. (dir), Sexualité et santé reproductive durant l'adolescence en Afrique, Boucherville, Ediconseil Inc., p. 133-152. 
98 African Population Studies Vol.20 n²/Etude de la population africaine vol. $20 n^{\circ} 2$

Lutz.W. : Sondage, Comment former les échantillons d'individus, de ménages, de zone, pour l'étude de zones de santé de la collectivité, École Nationale de Santé Publique, Rennes, France.

Meekers D. and Ahmed G. 1997. «Adolescent Sexuality in Southern Africa: Cultural Norms and Contemporary Behaviour ", Washington D.C., Population services international, Research Division, $28 \mathrm{p}$.

Meekers D. and Calvès A.E. 1997. "Gender Differentials in Adolescent Sexual Activity and Reproductive Health Risk in Cameroon », Washington D.C., Population services international, Research Division, $50 \mathrm{p}$.

Meekers D. 1994. "Sexual Initiation and Premarital Chlidbearing In Sub-Saharan Africa », in Population Studies, vol. 48, p. 47-64.

Moses Kamya Wiliam Mc Farland and al. Condom Use with Casual Partners by Men in Kampala Uganda in rapid science publishers ISSN 1350-2840.

Nations Unies. 1988. «Adolescent Sexual Behaviour: Evidence from Developing Countries ", in Population Studies, vol. II, 109 p.

Network. 1994. Les adolescents, Family Health International, Volume 9 Numéro 1.

NGUEYAP F. 1995. Ethnie, milieu d'habitat et propagation du HIV/SIDA. Évaluation, facteurs associés et implications. (Communication présentée au séminaire sur « Les aspects socio-économiques, sanitaires et démographiques du HIV/SIDA en Afrique ", Abidjan, 26-28 octobre NJIKAM SAVAGE O.M. (1998), "Croyances et perceptions des adolescents en matière de sexualité dans les zones urbaines du Cameroun", in KuATE-Defo B. (dir), Sexualité et santé reproductive durant l'adolescence en Afrique, Boucherville, Ediconseil Inc., p. 81-117.

Nzyuko S. Lurie P. and AL: Adolescent Sexual Behavior all the Trans-Africa Highway in Kenya in rapid science Publishers ISSN 1350.

Oms. 1998. Santé reproductive. Stratégie de la région africaine 1998-2007, Harare (Zimbabwe), Bureau régional de l'OMS pour l'Afrique, $23 \mathrm{p}$.

Oms/Unicef/Fnuap. 1997. Action en faveur des adolescents: vers un plan d'action, $15 \mathrm{p}$.

Onusida. 2001. Réduire la vulnérabilité des filles au VIH/SIDA, Meilleures pratiques, $57 \mathrm{p}$.

Oronsaye A. Odiasse. 1983. Attitudes toward Abortion and Contraception among Nigerian Secondary School Girls, International journal of Gyneacology and bstetrics. Vol. 21, n5 pp 423-426 October.

République Centrafricaine. 1995. Enquête Démographique et de Santé, 1995, Macro International inc.

Robinson N.J. et al. 1997. "Proportion of VIH Attributable to other Sexually Trasmitted Deseases in Rural Uganda Population: Simulation Model Estimates ", in International Journal of epidemiology, vol. 26, p. 180-189.

Rwenge M. 1995. Statut de la femme, comportements sexuels et SIDA en Afrique subsaharienne: le cas du Cameroun (Communication présentée au séminaire sur " les aspects socio-économiques, sanitaires et démographiques du VIH/SIDA en Afrique », organisé par l'UEPA, Abidjan, 26-28 octobre 1995).

Rwenge M. 1999. Facteurs contextuels des comportements sexuels: le cas des jeunes de la ville de Bamenda (Cameroun, Yaoundé, IFORD, UEPA, 164 p.

Rwenge M. 2000. "Sexual Risk Behaviors among Young People In Bamenda, Cameroon », International Family Planning Perspectives, vol. 26, n³ 3. p. 118-123.

Sehonou J. 1996. Mise au point 1996, le SIDA en Afrique Noire, Bangui.

Sehonou J. Grezenguet G. Donod ${ }^{\circledR}$ et al. 1999. Anonymous Voluntary HIV Counselling and Lessing in Central African Republic, Communition à la XIème ISACA (Lusaka), 12-16 Septembre.

Spencer B. 1993. "Contexte normatif du comportement sexuel et stratégies de prévention ", in Population, $\mathrm{n}^{\circ} 5$.

Standing H. and Kisekka M. 1989. Sexual Behaviour in Sub-Saharan Africa: A Review and Annoted Bibliography, London, Oversea Department. 
Susheela S. and Renee S. 1997. «Mariage précoce des femmes dans les pays en voie de dévelppement ", in Perspectives Internationales sur le Planning Familial, p. 4-14.

Twa-Twa J.M. 1997. "The Role of the Environment in Sexual Activity of School Students in Tororo and Pallisa Districts of Uganda », in Health Transition Review, Supplement to vol. 7, p. 67-81.

Udry J.R. 1986. "Biosocial Foundation for Adolescent Female Sexuality", in Demography, vol. 23, n², p. 217-226.

Van Rossen R. and Meekers D. 1999. An Evaluation of Effectiveness of Targeted Social Marketing to Promote Adolescent Young Adult Reproductive Health in Cameroon, Washington D.C., Population Service International, Research Division, Working paper, $32 \mathrm{p}$.

Zanele M. 1998. «Teenage Contraceptive Needs in Urban South Africa: A Case Study » in Perspectives Internationales sur le Planning Familial, vol. 24, n 4, p. 181 183.

Panos. 1993. Le vrai cô̂t du SIDA. Un nouveau défi au développement, Paris, l'Harmattan, $237 \mathrm{p}$.

Quan Zhou. 1996. "Childhood to Adulthood: A Difficult Transition", in UNFPA, International Youth Essay Contest: Promoting Responsible Reproductive Health Behaviour, Québec, Quebecor Printing Graphique Couleur, p. 8-9. 